\title{
Rapid increase in cystic volume of an anaplastic astrocytoma misdiagnosed as neurocysticercosis: A case report
}

\author{
HONG-JIANG LI ${ }^{1}$, HONG-XIU HAN ${ }^{2}$ and DONG-FU FENG ${ }^{1}$ \\ Departments of ${ }^{1}$ Neurosurgery and ${ }^{2}$ Pathology, No. 9 People's Hospital, Shanghai Jiaotong University School of Medicine, \\ Shanghai 201999, P.R. China
}

Received April 17, 2015; Accepted July 20, 2016

DOI: $10.3892 / \mathrm{ol} .2016 .4986$

\begin{abstract}
Reports describing a rapid increase in the cystic volume of anaplastic astrocytoma (AA) in a short time frame are rare. The present study reports the case of a 68-year-old male who was admitted to the No. 9 People's Hospital, Shanghai Jiaotong University School of Medicine (Shanghai, China), with a small cystic brain lesion and positive immunological testing for cysticercosis. Head magnetic resonance imaging (MRI) showed a cystic lesion, $6 \mathrm{~mm}$ in diameter, in the left frontal lobe. Neurocysticercosis was suspected and the patient was treated with a clinical trial of albendazole and steroids. A period of 25 days later, the patient's condition had deteriorated, and MRI revealed a cystic lesion in the left frontal lobe; thereafter, the cystic lesion was removed and a diagnosis of AA was established. The tumor was soft, ivory white and gelatinous due to myxoid degeneration. In this case, tumor-related angiogenesis and microvascular extravasation (blood-brain barrier disruption) may have been the main cause of the rapid increase in the cystic volume in such a short time frame. The similarity of the glioma and cysticercus antigens may have been the cause of the positive reactions in the cystic fluid. The present study reports the rare occurrence of a rapid increase of cystic volume and potential diagnostic difficulties.
\end{abstract}

\section{Introduction}

Anaplastic astrocytomas (AAs) are categorized as grade III tumors according to the 2007 World Health Organization (WHO) classification of tumors of the central nervous system (1). AAs are diffusely infiltrating tumors, commonly encountered as malignant primary central nervous system tumors in adults. The overall annual incidence of AA is an age-adjusted rate of 3.5 cases per million individuals (2). It has been reported that

Correspondence to: Professor Dong-Fu Feng, Department of Neurosurgery, No. 9 People's Hospital, Shanghai Jiaotong University School of Medicine, 280 Mohe Road, Baoshan, Shanghai 201999, P.R. China

E-mail: feng_df@yahoo.com

Key words: anaplastic astrocytoma, myxoid degeneration, cyst, immunological diagnosis, neurocysticercosis tumor-related angiogenesis and microvascular extravasation due to blood-brain barrier (BBB) disruption are the driving forces of cyst formation (3-5). Neurocysticercosis (NCC) is an infectious disease of the nervous system caused by the Taenia solium larvae (cysticerci) (6-8). Contaminated food and water are the major sources of this infection, causing severe headache and seizures in addition to pathological manifestations (9). An association between NCC and brain tumors has been reported (10-13) and may represent more than just a coincidence. However, reports on a rapid increase in the cystic volume of an AA in a short time frame (25 days) do not exist. The present study reports the case of a 68-year-old male with a positive immunological test result for cysticercosis in the serum, as assessed by enzyme-linked immunosorbent assay (ELISA), and an AA, for which the cystic volume increased rapidly from 6.5 to $37.5 \mathrm{~mm}$ in diameter within 25 days.

\section{Case report}

Presentation. A 68-year-old male was admitted to the No. 9 People's Hospital, Shanghai Jiaotong University School of Medicine (Shanghai, China) with a 1-month history of progressive weakness of the right lower limb followed by headaches. Head magnetic resonance imaging (MRI) showed a cystic lesion, $6 \mathrm{~mm}$ in diameter, in the left frontal lobe. The lesion was hypointense on axial T1-weighted images, hyperintense on axial T2-weighted images and slightly hyperintense on fluid-attenuated inversion recovery (FLAIR). The single small cyst had a relatively smooth, rim-enhancing lining on axial contrast-enhanced T1-weighted images (Fig. 1).

Treatment. The immunological testing in the serum by ELISA for cysticercosis was positive and NCC was suspected, leading to the patient being enrolled in a clinical trial with $400 \mathrm{mg}$ Albendazole twice per day for 10 days and $40 \mathrm{mg}$ steroids twice daily for 5 days. Initially, the symptoms improved. However, 25 days later, the patient was hemiplegic on the right side, and dysarthria was detected. MRI revealed a cystic lesion, $37 \mathrm{~mm}$ in diameter, in the left frontal lobe. The mass was hypointense on axial T1-weighted images, hyperintense on axial T2-weighted images and slightly hyperintense on FLAIR. The single large cyst exhibited a relatively smooth, rim-enhancing lining on axial contrast-enhanced T1-weighted images (Fig. 2). A left fronto-temporo-parietal craniotomy was performed, and the 


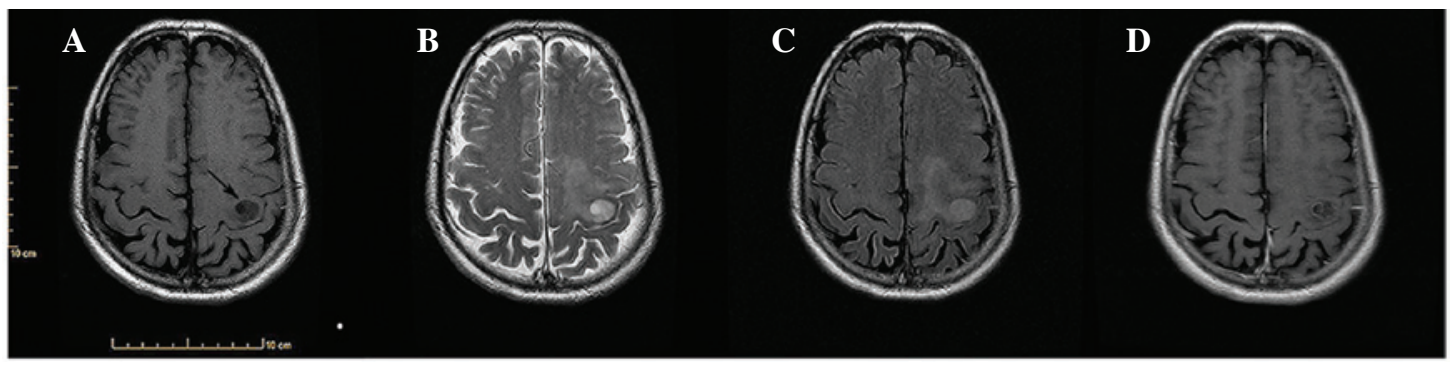

Figure 1. Magnetic resonance imaging on patient presentation showing a lesion (arrow) that was (A) hypointense on axial T1-weighted images, (B) hyperintense on axial T2-weighted images and (C) slightly hyperintense on fluid-attenuated inversion recovery. (D) The single small cyst exhibited a relatively smooth, rim-enhancing lining on axial contrast-enhanced T1-weighted images.

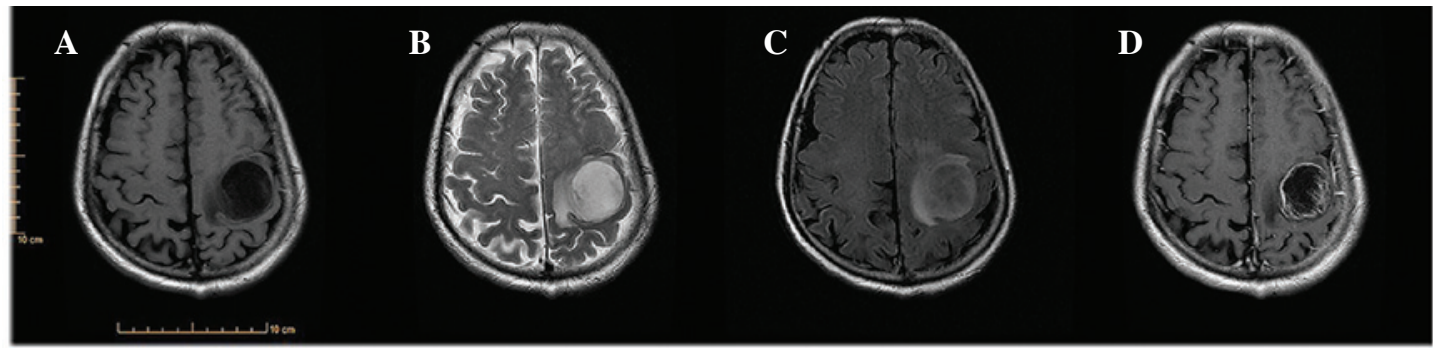

Figure 2. Magnetic resonance imaging at 25 days after the initiation of treatment showing a mass that was (A) hypointense on axial T1-weighted images, (B) hyperintense on axial T2-weighted images and (C) slightly hyperintense on fluid-attenuated inversion recovery. (D) The single large cyst exhibited a relatively smooth, rim-enhancing lining on axial contrast-enhanced T1-weighted images.
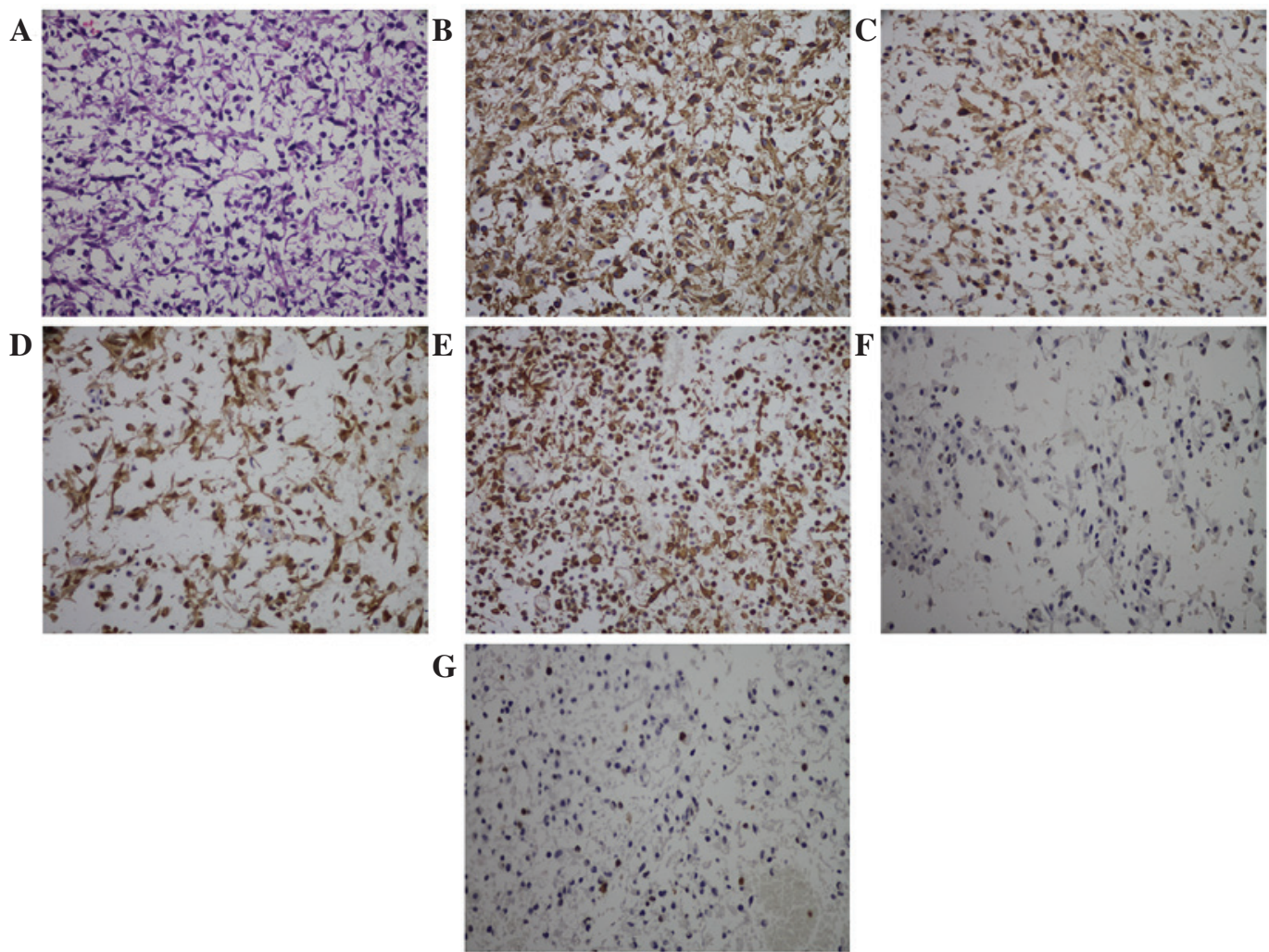

Figure 3. (A) Anaplastic astrocytoma, as shown by cells resembling astrocytes with hyperchromasia and nuclear pleomorphism (hematoxylin and eosin staining; original magnification, x200). Immunohistochemical studies positive for (B) glial fibrillary acidic protein, (C) epidermal growth factor receptor, (D) S100 and (E) vimentin, with $3 \%$ of cells positive for (F) p53 expression and 5\% positive for (G) Ki-67 expression in the fragments examined (original magnification, $\mathrm{x} 400$ ).

lesion was resected for intraoperative histological examination. During the lesion removal, the patient experienced a massive fluid flow from inside the lesion. The tissue was fixed in formalin and embedded in paraffin. Sections (5- $\mu$ m thick) were cut and stained with hematoxylin-eosin. Microscopic examination showed a relatively well-circumscribed tumor composed 
of structures resembling astrocytes with hyperchromasia and prominent nuclear pleomorphism. The diagnosis was of a malignant tumor. The tumor lesion was soft, ivory white and gelatinous. The tumor was completely removed during the surgery.

Post-operative pathological findings. Post-operative pathological investigations provided the diagnosis of AA, with cells resembling astrocytes with hyperchromasia and nuclear pleomorphism. Myxoid degeneration was observed, while vascular endothelial hyperplasia was absent (Fig. 3A). Immunohistochemical studies were positive for glial fibrillary acidic protein (Fig. 3B), epidermal growth factor receptor (Fig. 3C), S100 (Fig. 3D) and vimentin (Fig. 3E), and p53 (Fig. 3F) expression was determined in 3\% and Ki-67 (Fig. 3G) in 5\% of the fragments examined. An exhaustive search did not reveal any areas of inflammatory reaction in the specimens examined.

Post-operative course. The post-operative course was uneventful, and the weakness of the right limbs and dysarthria improved gradually. However, the patient refused further radiotherapy and chemotherapy. The patient was discharged at 11 days post-surgery and succumbed 7 months after the diagnosis.

\section{Discussion}

With regard to cyst formation, certain studies refer to the role of BBB disruption in tumor cyst formation, based on the chemical assessment of high plasma protein concentrations ( $92 \%$ of the total protein) in the cysts, without evidence of bleeding $(5,14)$. Tumor-related angiogenesis and microvascular extravasation (BBB disruption) are activated and mediated by polypeptides, including vascular endothelial growth/permeability factor, and are thus the driving forces of cyst formation (3-5). Resolution of brain edema is believed to occur via drainage into the cerebrospinal spaces and by the degradation of extravasated plasma fluid and proteins by proteolytic activity $(14,15)$. Cyst formation as a result of a deficient resolution mechanism should therefore be considered.

The cystic volume of the present patient increased rapidly and the lesion was gelatinous. The post-operative pathological result was of AA, myxoid degeneration, WHO grade III. AA aggregating into gelatinous material and a rapid increase in cystic volume within a short time frame (25 days), as shown in the present study, have not previously been found. We believe that tumor-related angiogenesis and microvascular extravasation (BBB disruption) may be the main cause of the rapid increase of cystic volume. However, further research is required to determine whether myxoid degeneration can promote cystic formation.

The current patient also presented with a cystic brain lesion and an immunological positive reaction for cysticercosis. The diagnosis of an AA was established after examining the specimens obtained by surgery. In a retrospective analysis of these histopathological specimens, no inflammatory reaction was found. Positive immunological testing for cysticercosis in the cystic fluid of gliomas was reported for the first time by Agapejev et al (16). These findings are similar to those reported by Salomão (10), who also described a positive reaction for cysticercosis and multicentric anaplastic oligoastrocytoma, with no inflammatory reaction surrounding the cystic wall. In our opinion, the possibility of a tumor developing from an unapparent parasitic lesion is invalidated by this finding. As stated by Salomão et al (10), the glioma and cysticercosis antigens are glycoproteins of a similar molecular weight, which may be the cause of the positive reaction for NCC in the cystic fluid.

In conclusion, reports describing a rapid increase in the cystic volume of AA in a short time frame were rare prior to the current literature. In the present case, tumor-associated angiogenesis and microvascular extravasation (blood-brain barrier disruption) may have been the major cause of the rapid increase in the cystic volume in such a short time frame. Cystic AA can be easily misdiagnosed as neurocysticercosis merely based on clinical symptoms, MRI and the immunological testing due to the similarity of the glioma and cysticercus antigens. Pathological investigations following surgical treatment are vital for the management of this condition.

\section{References}

1. Louis DN, Ohgaki H, Wiestler OD, Cavenee WK, Burger PC, Jouvet A, Scheithauer BW and Kleihues P: The 2007 WHO classification of tumours of the central nervous system. Acta Neuropathol 114: 97-109, 2007.

2. Smoll NR and Hamilton B: Incidence and relative survival of anaplastic astrocytomas. Neuro Oncol 16: 1400-1407, 2014.

3. Strugar JG, Criscuolo GR, Rothbart D and Harrington WN: Vascular endothelial growth/permeability factor expression in human glioma specimens: Correlation with vasogenic brain edema and tumor-associated cysts. J Neurosurg 83: 682-689, 1995.

4. Lohle PN, van Mameren H, Zwinderman KH, Teepen HL, Go KG and Wilmink JT: On the pathogenesis of brain tumour cysts: A volumetric study of tumour, oedema and cyst. Neuroradiology 42 : 639-642, 2000

5. Weindel K, Moringlane JR, Marmé D and Weich HA: Detection and quantification of vascular endothelial growth factor/vascular permeability factor in brain tumor tissue and cyst fluid: The key to angiogenesis? Neurosurgery 35: 439-449, 1994.

6. Pittella JE: Neurocysticercosis. Brain Pathol 7: 681-693, 1997.

7. Caplan LR: How to manage patients with neurocysticercosis. Eur Neurol 37: 124, 1997.

8. Sabel M, Neuen-Jacob E, Vogt C and Weber F: Intracerebral neurocysticercosis mimicking glioblastoma multiforme: A rare differential diagnosis in Central Europe. Neuroradiology 43: 227-230, 2001.

9. Rizvi SA, Saleh AM, Frimpong H, Al Mohiy HM, Ahmed J, Edwards RD and Ahmed SS: Neurocysticercosis: A case report and brief review. Asian Pac J Trop Med 9: 100-102, 2016.

10. Salomão JF, Pone MV, da Silva AR, Leibinger RD, Bellas AR, Campos JM, Garrido JR, Vanazzi E, de Barros AC, Pone SM and Boechat MB: Positive reaction for cysticercosis and multicentric anaplastic oligoastrocytoma. Childs Nerv Syst 22: 182-185, 2006.

11. Tripathi RP, Gupta A, Gupta S, Kumaran SS, Khushu S, Dev A and Balwant: Co-existence of dual intracranial pathology clinical relevance of proton MRS. Neurol India 48: 365-369, 2000.

12. Hautecoeur P, Gallois P, Brucher JM, Ovelacq E and Dereux JF: Association of cerebral cysticercosis and multifocal glioma. Discussion of interactions. Rev Neurol (Paris) 143: 844-849, 1987 (In French).

13. Lohle PN, Verhagen IT, Teelken AW, Blaauw EH and Go KG: The pathogenesis of cerebral gliomatous cysts. Neurosurgery 30 : 180-185, 1992.

14. Lohle PN, Wurzer HA, Seelen PJ, Kingma LM and Go KG: The pathogenesis of cysts accompanying intra-axial primary and metastatic tumors of the central nervous system. J Neurooncol 40: 277-285, 1998.

15. Bodsch W and Hossmann KA: 125I-antibody autoradiography and peptide fragments of albumin in cerebral edema. J Neurochem 41 : 239-243, 1983

16. Agapejev S, Alves A, Zanini MA, Ueda AK and Pereira EM: Cystic oligodendroglioma and positivity of reactions for cysticercosis: Report of a case. Arq Neuropsiquiatr 50: 234-238, 1992 (In Portuguese). 